AperTO - Archivio Istituzionale Open Access dell'Università di Torino

\title{
Coendomorphism left bialgebroids
}

\section{This is the author's manuscript}

Original Citation:

Availability:

This version is available http://hdl.handle.net/2318/102928

since

Published version:

DOI:10.1142/S0219498812501812

Terms of use:

Open Access

Anyone can freely access the full text of works made available as "Open Access". Works made available under a Creative Commons license can be used according to the terms and conditions of said license. Use of all other works requires consent of the right holder (author or publisher) if not exempted from copyright protection by the applicable law. 


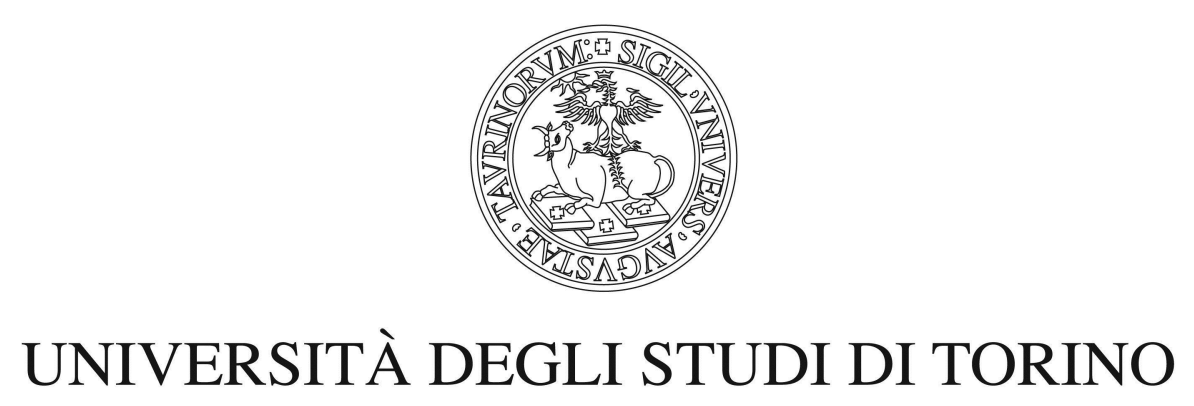

This is an author version of the contribution published on:

Alessandro ARDIZZONI, Laiachi EL KAOUTIT and Claudia MENINI. Coendomorphism left bialgebroids. Journal of Algebra and Its Applications, Vol. 12, No. 3 (2013) 1250181 (21 pages). DOI: 10.1142/S0219498812501812

The definitive version is available at:

http://www.worldscientific.com/doi/abs/10.1142/S0219498812501812 


\title{
COENDOMORPHISM LEFT BIALGEBROIDS
}

\author{
A. ARDIZZONI, L. EL KAOUTIT, AND C. MENINI
}

\begin{abstract}
The main purpose of this note is to give a rigorous proof of the construction of coendomorphism left bialgebroids as well as an explicit description of their structure maps. We also compute some concrete examples of these objects by means of their generators and relations.
\end{abstract}

\section{INTRODUCTION}

In recent decades the notion of Hopf algebroid, both in the commutative and noncommutative case, appeared as an important tool in different branches of pure mathematics: algebraic topology, abstract algebraic geometry (Tannaka-Krein duality), Poisson geometry, Lie groupoids and Lie algebroids, see for instance $[9,5,4,7,6]$.

While commutative Hopf algebroids can be directly defined as presheaves of groupoids in affine schemes, the definition of noncommutative Hopf algebroid behaves in much more complicated way. In categorical terms [10, 11], a left Hopf algebroid can be thought as a ring extension of the enveloping ring of the base algebra, whose category of left modules is a right closed monoidal category and the canonical forgetful functor to the category of bimodules (over the base algebra) is strict monoidal and preserves right inner hom-functors. It is worth mentioning that in practice the most difficult task in checking these properties is to verify this last condition. Dropping it from the definition one obtains the notion of left bialgebroid.

There certainly exists a strong motivation in these areas for studying the structure of Hopf algebroids, but there is one basic problem especially concerning the noncommutative ones which turns out to be of much more fundamental nature. Namely, the lack of examples of left Hopf algebroids or at least left bialgebroids that can be defined by means of generators and relations. Moreover, from the noncommutative algebraic geometry point of view, the construction of this kind of left bialgebroids certainly is the most desirable.

The main aim of this note is to establish in a rigorous way the construction of the coendomorphism left bialgebroids and give concrete examples by means of their generators and relations, hoping by this to fulfil the lack of examples in the theory of bialgebroids. Specifically, we start with an extension of $\mathbb{k}$-algebras $R \rightarrow A$ ( $\mathbb{k}$ is any commutative ground

Date: February 20, 2014.

2010 Mathematics Subject Classification. Primary 16D90, 16T05; Secondary 18D10, 16 T15.

Key words and phrases. Monoidal categories; Ring extension; Bialgebroids.

This paper was written while the first and the last authors were members of GNSAGA. The second author was supported by grant MTM2010-20940-C02-01 from the Ministerio de Educación y Ciencia of Spain. His stay, as a visiting professor at University of Ferrara, was supported by INdAM. 
ring with 1) and assume that ${ }_{R} A$ is a finitely generated and projective left module. We then consider the monoidal functor $-\times_{R} A$ from the category of $\left(R \otimes_{\mathbb{k}} R^{o}\right)$-bimodules to the category of $R$-bimodules, where $-\times_{R}-$ is Sweedler-Takeuchi's product $[12,14]$ and $R^{o}$ denotes the opposite algebra of $R$. Since $-\times_{R} A$ has a left adjoint and can be lifted to a functor from the category of $\left(R \otimes_{\mathbb{k}} R^{o}\right)$-rings to the category of $R$-rings, using a classical monoidal result, we know that there is a left adjoint functor of this lifting which we denoted by $\mathscr{L}_{m}$. In the first section we give an explicit description of the functor $\mathscr{L}_{m}$ and prove that the image $\mathscr{L}_{m}(A)$ of $A$, admits a structure of left bialgebroid such that $A$ becomes a left comodule $R$-ring. It is worth noting that the possibility of this construction was first observed by D. Tambara in [15, Remark 1.7] where no proof was provided. Several concrete examples of coendomorphism left bialgebroids including some new coendomorphism bialgebras, are also given in the second section.

Basic notions and notations. We work over a ground commutative ring with 1 denoted by $\mathbb{k}$. All rings under consideration are $\mathbb{k}$-algebras, and morphisms of rings are morphisms of $\mathbb{k}$-algebras. Modules are assumed to be unital modules and bimodules are assumed to be central $\mathbb{k}$-bimodules. For every ring $R$, we will denote by ${ }_{R} \operatorname{Mod}, \operatorname{Mod}_{R}$ and ${ }_{R} \operatorname{Mod}_{R}$ the categories of left, right and two-sided modules over $R$ respectively. The tensor product over $R$, is denoted as usual by $-\otimes_{R}-$. The identity morphism of a right, left module or bimodule $M$ is denoted by the object itself $M$.

For two bimodules ${ }_{R} P_{S}$ and ${ }_{R} Q_{S}$ over rings $R$ and $S$, we will consider the $\mathbb{k}$-modules of $R$-linear maps $\operatorname{Hom}_{R-}(P, Q)$ as an $S$-bimodule with actions:

$s f: p \mapsto f(p s)$, and $f s^{\prime}: p \mapsto f(p) s^{\prime}$, for every $f \in \operatorname{Hom}_{R-}(P, Q), s, s^{\prime} \in S$, and $p \in P$.

Similarly, $\operatorname{Hom}_{-S}(P, Q)$ is considered as an $R$-bimodule with actions:

$r g: p \mapsto r g(p)$, and $g r^{\prime}: p \mapsto g\left(r^{\prime} p\right)$, for every $g \in \operatorname{Hom}_{-S}(P, Q), r, r^{\prime} \in R$, and $p \in P$.

Under these considerations, the left dual ${ }^{*} X=\operatorname{Hom}_{R-}(X, R)$ of a given $R$-bimodule $X$, is an $R$-bimodule, as well as its right dual $X^{*}=\operatorname{Hom}_{-R}(X, R)$. The sub $\mathbb{k}$-module of $R$-invariant elements of $X$ is denotes by:

$$
X^{R}:=\{x \in X \mid r x=x r, \forall r \in R\} \cong \operatorname{Hom}_{R-R}(R, X) .
$$

This in fact defines a functor from $R$-bimodules to the category of modules over the center algebra.

For a fixed ring $R$, we denote by $R$-Rings the category of $R$-rings. This is the comma category over $R$ in the category of all $\mathbb{k}$-algebras. That is, objects are morphisms of rings $R \rightarrow A$ and morphisms are commutative triangles of $\mathbb{k}$-algebra maps. Obviously, this category is identified with the category of monoids of the monoidal category of bimodules ${ }_{R} \operatorname{Mod}_{R}$. Dually, one can define $R$-corings [13]. Explicitly, an $R$-coring is a comonoid in ${ }_{R} \operatorname{Mod}_{R}$, which is by definition a three-tuple $(\mathfrak{C}, \Delta, \varepsilon)$ consisting of an $R$-bimodule $\mathfrak{C}$ and two $R$-bilinear maps $\Delta: \mathfrak{C} \rightarrow \mathfrak{C} \otimes_{R} \mathfrak{C}$ (comultiplication), $\varepsilon: \mathfrak{C} \rightarrow R$ (counit) satisfying the usual coassociativity and counitary constraints. 
For more information on corings and theirs comodules, the reader is referred to [2]. For the notions of bialgebroids and their basic properties, the reader is referred to [3].

\section{The CONSTRUCtion OF COENDOMORPHism BialgeBroids.}

The construction of the coendomorphism bialgebroids is performed in this section. We proceed as follows. Let $A$ be an $R$-ring and assume that ${ }_{R} A$ is finitely generated and projective. We first show that the monoidal functor $-\times_{R} A: R^{\mathrm{e}} \operatorname{Mod}_{R^{\mathrm{e}}} \rightarrow{ }_{R} \operatorname{Mod}_{R}$ has a left adjoint functor, where $R^{\mathrm{e}}=R \otimes_{\mathbb{k}} R^{o}$ is the enveloping ring and $-\times_{R}-$ is the SweedlerTakeuchi product $[12,14]$. This allows us to apply a classical result in monoidal categories theory (stated here as Theorem 1.3.4), in order to construct a functor $\mathscr{L}_{m}: R$-Rings $\rightarrow$ $R^{\mathrm{e}}$-Rings which is left adjoint to $-\times_{R} A: R^{\mathrm{e}}$-Rings $\rightarrow R$-Rings. We then prove that the image of $A$ under this functor, i.e. $\mathscr{L}_{m}(A)$ admits a structure of left $R$-bialgebroid such that $A$ becomes a left $\mathscr{L}_{m}(A)$-comodule.

1.1. An adjunction between $R^{\mathrm{e}}$-bimodules and $R$-bimodules. The unadorned symbol $\otimes$ stands for the tensor product over the commutative ground ring $\mathbb{k}$. Let $R$ be a ring. For any $r \in R$, we denote by $r^{o}$ the same element regarded as an element in the opposite ring $R^{o}$. Let $R^{\mathrm{e}}:=R \otimes R^{o}$ be the enveloping ring of $R$. Given an $R^{\mathrm{e}}$-bimodule $M$, the underlying $\mathbb{k}$-module $M$ admits several structures of $R$-bimodule. Among them, we will select the following two ones. The first structure is that of the opposite bimodule $1 \otimes R^{o} M_{1 \otimes R^{o}}$ which we denote by $M^{o}$. That is, the $R$-biaction on $M^{o}$ is given by

$$
r m^{o}=m\left(1 \otimes r^{o}\right), \quad m^{o} s=\left(1 \otimes s^{o}\right) m, \quad m^{o} \in M^{o}, r, s \in R .
$$

Notice, that this construction defines in fact a functor $(-)^{o}:{ }_{R^{\mathrm{e}}} \operatorname{Mod}_{R^{\mathrm{e}}} \rightarrow{ }_{R} \operatorname{Mod}_{R}$. The second structure is defined by the left $R^{\mathrm{e}}$-module $R_{R^{\mathrm{e}}} M$. That is, the $R$-bimodule $M^{l}=$ $R \otimes 1^{\circ} M_{R}$ whose $R$-biaction is defined by

$$
r m^{l}=\left(r \otimes 1^{o}\right) m, \quad m^{l} s=\left(1 \otimes s^{o}\right) m, \quad m^{l} \in M^{l}, r, s \in R .
$$

This also defines a functor, namely, the right $R^{\mathrm{e}}$-action forgetful functor $(-)^{l}:{ }_{R^{\mathrm{e}}} \mathrm{Mod}_{R^{\mathrm{e}}} \rightarrow$ ${ }_{R} \operatorname{Mod}_{R}$. One easily observes that there is a commutative diagram:

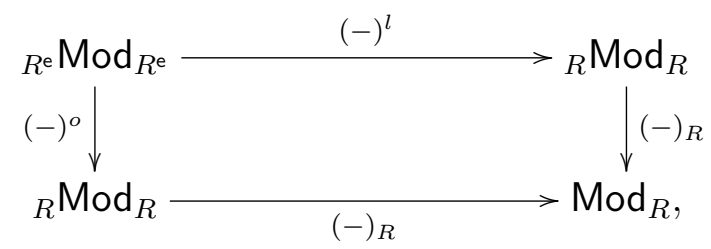

where $(-)_{R}$ is the left $R$-action forgetful functor.

Another $R^{\mathrm{e}}$-bimodule derived from $M$, which will be used in the sequel, is $M^{\dagger}$. The underlying $\mathbb{k}$-module of $M^{\dagger}$ is $M$ and an element $m \in M$ is denoted by $m^{\dagger}$ when it is viewed in $M^{\dagger}$. The $R^{\mathrm{e}}$-biaction on $M^{\dagger}$ is given by

$$
\left(p \otimes q^{o}\right) m^{\dagger}\left(r \otimes s^{o}\right)=\left(p \otimes r^{o}\right) m\left(q \otimes s^{o}\right), \quad m^{\dagger} \in M^{\dagger}, p, r \in R, q^{o}, s^{o} \in R^{o} .
$$


Here also we have a functor $(-)^{\dagger}:{ }_{R^{\mathrm{e}}} \operatorname{Mod}_{R^{\mathrm{e}}} \rightarrow{ }_{R^{\mathrm{e}}} \operatorname{Mod}_{R^{\mathrm{e}}}$ which has the following properties (6) $R^{\mathrm{e}}\left(M^{\dagger}\right)^{\dagger} R^{\mathrm{e}}=R^{\mathrm{e}} M_{R^{\mathrm{e}}} \quad$ and $\quad \operatorname{Hom}_{R^{\mathrm{e}}-R^{\mathrm{e}}}\left(M^{\dagger}, U^{\dagger}\right)=\operatorname{Hom}_{R^{\mathrm{e}}-R^{\mathrm{e}}}(M, U)$, for every pair of $R^{\mathrm{e}}$-bimodules $U$ and $M$. Furthermore, there is a commutative diagram

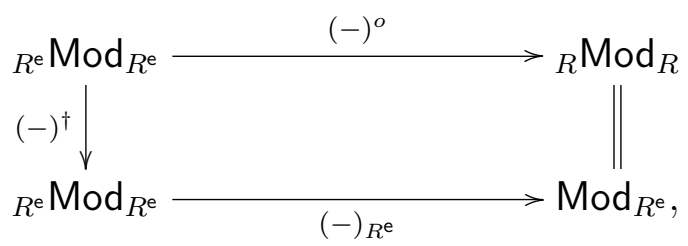

where as before $(-)_{R^{\text {e }}}$ denotes the left $R^{\mathrm{e}}$-action forgetful functor. It is clear that the left module $R^{\mathrm{e}} M^{\dagger}$ induces the already existing $R$-bimodule structure of $R \otimes 1^{\circ} M_{R \otimes 1^{o}}$.

Now, let $N$ be another $R$-bimodule, and consider the tensor product $M^{o} \otimes_{R} N$. The additive $\mathbb{k}$-submodule of invariant elements

$$
\left(M^{o} \otimes_{R} N\right)^{R}=\left\{\sum_{i} m_{i}^{o} \otimes_{R} n_{i} \mid \sum_{i} r m_{i}^{o} \otimes_{R} n_{i}=\sum_{i} m_{i}^{o} \otimes_{R} n_{i} r, \text { for all } r \in R\right\}
$$

admits a structure of an $R$-bimodule given by the actions:

$$
\begin{aligned}
r \rightarrow\left(\sum_{i} m_{i}^{o} \otimes_{R} n_{i}\right) & =\sum_{i}\left(\left(r \otimes 1^{o}\right) m_{i}\right)^{o} \otimes_{R} n_{i}, \\
\left(\sum_{i} m_{i}^{o} \otimes_{R} n_{i}\right)<s & =\sum_{i}\left(m_{i}\left(s \otimes 1^{o}\right)\right)^{o} \otimes_{R} n_{i},
\end{aligned}
$$

for every set of elements $\sum_{i} m_{i}^{o} \otimes_{R} n_{i} \in M^{o} \otimes_{R} N$ and $r, s \in R$.

In this way, to each $R$-bimodule $N$ one associates two functors:

$$
\left((-)^{o} \otimes_{R} N\right)^{R}:{ }_{R^{\mathrm{e}}} \operatorname{Mod}_{R^{\mathrm{e}}} \longrightarrow{ }_{R} \operatorname{Mod}_{R}, \quad\left(-\otimes^{*} N\right)^{\dagger}:{ }_{R} \operatorname{Mod}_{R} \longrightarrow{ }_{R^{\mathrm{e}}} \operatorname{Mod}_{R^{\mathrm{e}}},
$$

where, for each $R$-bimodule $X$, we consider $X \otimes{ }^{*} N$ as an $R^{\mathrm{e}}$-bimodule with the following actions

$$
\left(p \otimes q^{o}\right)\left(\sum_{i} x_{i} \otimes \varphi_{i}\right)\left(r \otimes s^{o}\right)=\sum_{i}\left(p x_{i} q\right) \otimes\left(s \varphi_{i} r\right),
$$

for every element $\sum_{i} x_{i} \otimes \varphi_{i} \in X \otimes{ }^{*} N, p, q, r, s \in R$. These functors are related as follows. LEMMA 1.1.1. Let $N$ be an R-bimodule such that ${ }_{R} N$ is finitely generated and projective module with left dual basis $\left\{\left(e_{j},{ }^{*} e_{j}\right)\right\}_{1 \leq j \leq m} \subset N \times{ }^{*} N$. There is a natural isomorphism

$$
\begin{gathered}
\operatorname{Hom}_{R-R}\left(X,\left(M^{o} \otimes_{R} N\right)^{R}\right) \longrightarrow \operatorname{Hom}_{R^{\mathrm{e}}-R^{\mathrm{e}}}\left(\left(X \otimes{ }^{*} N\right)^{\dagger}, M\right) \\
\sigma \longmapsto\left[(x \otimes \varphi)^{\dagger} \longmapsto\left(\left(M^{o} \otimes_{R} \varphi\right) \circ \sigma(x)\right)\right] \\
{\left[x \longmapsto \sum_{j} \alpha\left(\left(x \otimes^{*} e_{j}\right)^{\dagger}\right)^{o} \otimes_{R} e_{j}\right] \longleftrightarrow} \\
{[\alpha}
\end{gathered}
$$


for every R-bimodule $X$ and $R^{\mathrm{e}}$-bimodule $M$. Equivalently, the functor $\left(-\otimes^{*} N\right)^{\dagger}$ is left adjoint to the functor $\left((-)^{o} \otimes_{R} N\right)^{R}$.

Proof. By the isomorphism $\left(M^{o} \otimes_{R} N\right)^{R} \cong \operatorname{Hom}_{-R^{\mathrm{e}}}\left(R, M^{o} \otimes_{R} N\right)$ of $\mathbb{k}$-modules, the righthand object inherits a structure of left $R^{\mathrm{e}}$-module coming from the actions $\rightarrow,<$ defined in (8) and (9). This left $R^{\mathrm{e}}$-action is explicitly given by the formula:

$$
\left(\left(p \otimes q^{o}\right) \alpha\right)(1)=p \rightarrow \alpha(1)<q,
$$

for every $p, q \in R$, and $\alpha \in \operatorname{Hom}_{-R^{\mathrm{e}}}\left(R, M^{o} \otimes_{R} N\right)$. Since ${ }_{R} N$ is finitely generated and projective, we have a $\mathbb{k}$-linear isomorphism

$\mathrm{f}: \operatorname{Hom}_{-R^{\mathrm{e}}}\left(R, M^{o} \otimes_{R} N\right) \stackrel{\cong}{\longrightarrow} \operatorname{Hom}_{-R^{\mathrm{e}}}\left({ }^{*} N, M^{\dagger}\right), \quad\left(\alpha \longmapsto\left[\varphi \mapsto\left(M^{o} \otimes_{R} \varphi\right) \circ \alpha(1)\right]\right)$, with inverse map $\mathrm{f}^{-1}(\sigma)(1)=\sum_{j} \sigma\left({ }^{*} e_{j}\right)^{o} \otimes_{R} e_{j}$, for every $\sigma \in \operatorname{Hom}_{-R^{\mathrm{e}}}\left({ }^{*} N, M^{\dagger}\right)$ (recall that the underlying right $R^{\mathrm{e}}$-module of the $R^{\mathrm{e}}$-bimodule $M^{\dagger}$ is $M^{o}$, see diagram (7)). One can show that $\mathrm{f}$ is left $R^{\mathrm{e}}$-linear, where $\operatorname{Hom}_{-R^{\mathrm{e}}}\left({ }^{*} N, M^{\dagger}\right)$ is a left $R^{\mathrm{e}}$-module by the $R^{\mathrm{e}}$-bimodule structure of $M^{\dagger}$. We then obtain the following chain of natural isomorphisms

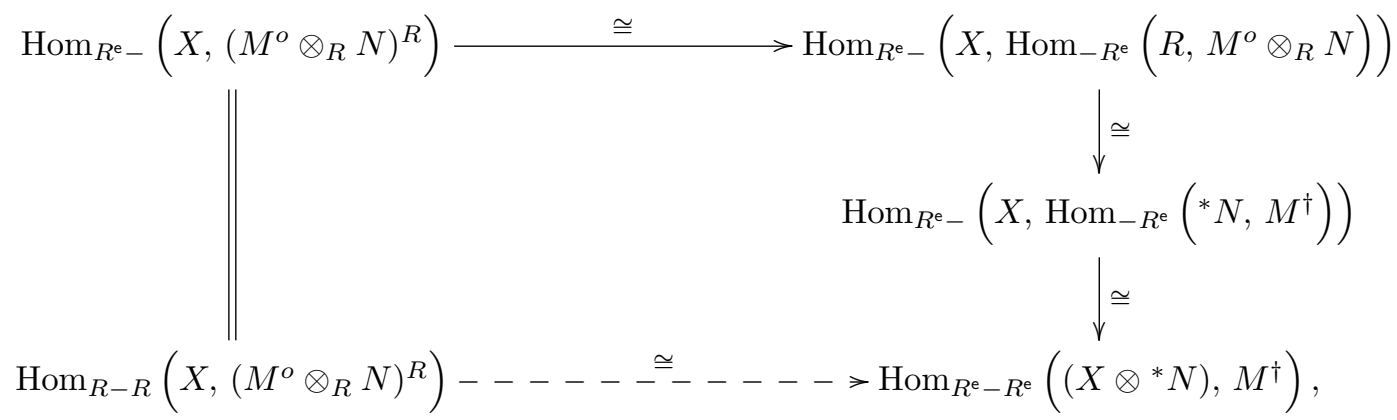

where the second right vertical isomorphism is the usual Tensor-Hom adjunction. Since by (6) the functor $(-)^{\dagger}$ is self-adjoint, the right-hand term in the second row is isomorphic to the $\mathbb{k}$-module $\operatorname{Hom}_{R^{\mathrm{e}}-R^{\mathrm{e}}}\left(\left(X \otimes{ }^{*} N\right)^{\dagger}, M\right)$ so that we get the desired natural isomorphism.

1.2. The bi-functor $\left(-\times_{R}-\right)$ on $R^{\mathrm{e}}$-bimodules. For the convenience of the reader who is not familiar with the notion of $\times_{R}$-bialgebras [14], [10], we give in this subsection a detailed definition of these objects. The most difficult part is the specification of the bi-functor $-\times_{R}-$ and their domain and co-domain categories. Here we substitute Mac Lane's functors $\int_{x}, \int^{y}$ (end, coend) by the tensor product $-\otimes_{R}-$ and the "invariants" $(-)^{R}$ functors of Eq (1), which we believe is much closer to the usual notation in bimodules theory.

As we have seen in Subsection 1.1, there is a bi-functor

$$
-\times_{R}-:=\left((-)^{o} \otimes_{R}-\right)^{R}:{ }_{R} \operatorname{Mod}_{R^{\mathrm{e}}} \times{ }_{R} \operatorname{Mod}_{R} \longrightarrow{ }_{R} \operatorname{Mod}_{R} .
$$

This is Sweedler-Takeuchi's product of bimodules [12], [14], which can be also defined using the notion of ends (limits) and coends (colimits), see [8, pages 222 and 226]. 
Given an $R^{\mathrm{e}}$-bimodule $M$ and an $R$-bimodule $N$, an element $\sum_{i} m_{i}^{o} \otimes_{R} n_{i}$ which belongs to $M \times_{R} N$ will be denoted by $\sum_{i} m_{i} \times_{R} n_{i}$. Thus, for every $r \in R$ and $m \times_{R} n \in M \times_{R} N$, we have

$$
\left(m\left(1 \otimes r^{o}\right)\right) \times_{R} n=m \times_{R} n r, \text { and }\left(\left(1 \otimes r^{o}\right) m\right) \times_{R} n=m \times_{R} r n .
$$

With this notation the left $R^{\mathrm{e}}$-action on $M \times_{R} N$ defined in (8) and (9) can be written as follows:

$$
\left(r \otimes s^{o}\right)\left(\sum_{i} m_{i} \times_{R} n_{i}\right)=\sum_{i}\left(r \otimes 1^{o}\right) m_{i}\left(s \otimes 1^{o}\right) \times_{R} n_{i},
$$

for every elements $\sum_{i} m_{i} \times_{R} n_{i} \in M \times_{R} N$ and $r, s \in R$.

Next, we want to restrict the bi-functor $\left(-\times_{R}-\right)$ to the product category of $R^{\mathrm{e}}$-bimodules $R^{\mathrm{e}} \operatorname{Mod}_{R^{\mathrm{e}}} \times R_{R^{\mathrm{e}}} \operatorname{Mod}_{R^{\mathrm{e}}}$. As one can realize there are many ways to do it. That is, if $N$ is an $R^{\mathrm{e}}$-bimodule, then there are several structures of $R$-bimodules on $N$ over which one can construct $M \times_{R} N$. Here we define $M \times_{R} N$ by using the $R$-bimodule $R \otimes 1^{\circ} N_{R \otimes 1^{o}}$.

In this way, $M \times{ }_{R} N$ admits a structure of $R^{\mathrm{e}}$-bimodule: Using the above left $R^{\mathrm{e}}$-action (11), we obtain an $R^{\mathrm{e}}$-biaction

$$
\left(r \otimes s^{o}\right)\left(\sum_{i} m_{i} \times_{R} n_{i}\right)\left(p \otimes q^{o}\right)=\sum_{i}\left(\left(r \otimes 1^{o}\right) m_{i}\left(s \otimes 1^{o}\right)\right) \times_{R}\left(\left(1 \otimes p^{o}\right) n_{i}\left(1 \otimes q^{o}\right)\right),
$$

for every elements $\sum_{i} m_{i} \times_{R} n_{i} \in M \times_{R} N$ and $r, s, p, q \in R$. Hence the $R^{\mathrm{e}}$-biaction on $\left(M \times_{R} N\right)^{\dagger}$ is given by the formula:

$$
\left(r \otimes s^{o}\right)\left(\sum_{i} m_{i} \times_{R} n_{i}\right){ }^{\dagger}\left(p \otimes q^{o}\right)=\left(\sum_{i}\left(\left(r \otimes 1^{o}\right) m_{i}\left(p \otimes 1^{o}\right)\right) \times_{R}\left(\left(1 \otimes s^{o}\right) n_{i}\left(1 \otimes q^{o}\right)\right)\right){ }^{\dagger} .
$$

¿From now on, the restriction of the bi-functor $\left(-\times_{R}-\right)$ to $R_{R^{\mathrm{e}}} \operatorname{Mod}_{R^{\mathrm{e}}} \times{ }_{R^{\mathrm{e}}} \operatorname{Mod}_{R^{\mathrm{e}}}$ will be understood as the following compositions of functors:

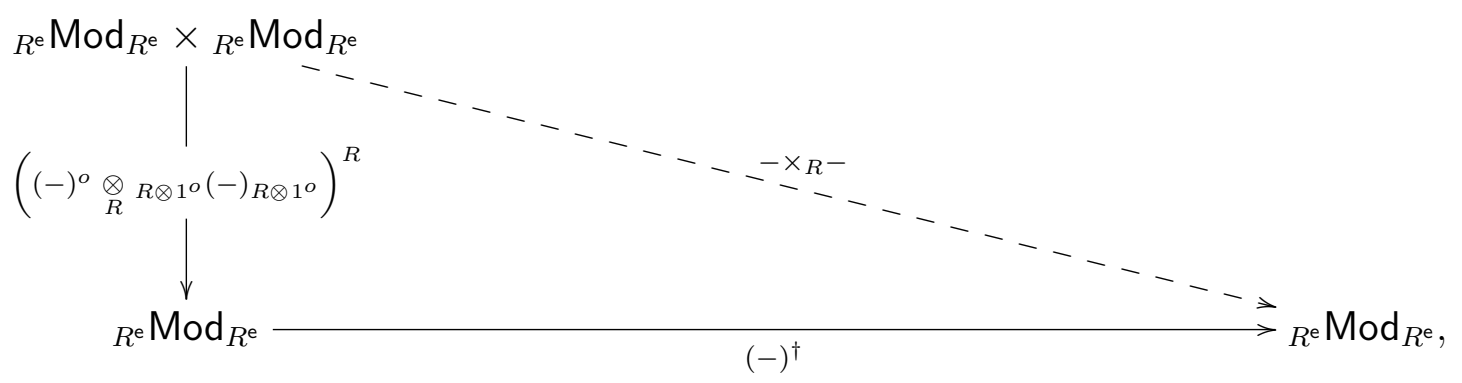

and this will be our definition for $\times_{R}$-product of $R^{\mathrm{e}}$-bimodules. Therefore, for two bimodules $R^{\mathrm{e}} M_{R^{\mathrm{e}}}$ and ${ }_{R^{\mathrm{e}}} N_{R^{\mathrm{e}}}$, we set

$$
M \times_{R} N:=\left[\left(\mathbf{M} \otimes_{R} \mathbf{N}\right)^{R}\right]^{\dagger},
$$


where ${ }_{R} \mathbf{M}_{R}=\left({ }_{1 \otimes R^{o}} M_{1 \otimes R^{o}}\right)^{o}$ and ${ }_{R} \mathbf{N}_{R}={ }_{R \otimes 1^{o}} N_{R \otimes 1^{o}}$. Thus, (10) reads as

$$
\left(m\left(1 \otimes r^{o}\right)\right) \times_{R} n=m \times_{R} n\left(r \otimes 1^{o}\right), \text { and }\left(\left(1 \otimes r^{o}\right) m\right) \times_{R} n=m \times_{R}\left(r \otimes 1^{o}\right) n,
$$

and (13) as

$$
\left.\left(p \otimes q^{o}\right) m \times_{R} n\left(r \otimes s^{o}\right)=\left(\left(p \otimes 1^{o}\right) m\left(r \otimes 1^{o}\right)\right) \times_{R}\left(\left(1 \otimes q^{o}\right) n\left(1 \otimes s^{o}\right)\right)\right),
$$

for every $r, s, p, q \in R$ and $m \times_{R} n \in M \times_{R} N$.

On the other hand, since we have $M_{R}^{o}=M_{R}^{l}$ for every $R^{\mathrm{e}}$-bimodule $M$, there is a canonical natural transformation (which is injective)

$$
\Theta_{M, N}: M \times_{R} N \longrightarrow M^{l} \otimes_{R} N^{l} .
$$

Now, given another $R^{\mathrm{e}}$-bimodule $W$, there are three $R^{\mathrm{e}}$-bimodules under consideration. Namely, $M \times_{R}\left(N \times_{R} U\right),\left(M \times_{R} N\right) \times_{R} U$, and $M \times_{R} N \times_{R} W$. The later is constructed as follows: First we consider the underlying left $R^{\mathrm{e}}$-module of $N$, that is, $N^{l}={ }_{R^{\mathrm{e}}} N$ which we consider obviously as an $R$-bimodule, see diagram (4). Secondly, we construct the $\mathbb{k}$ module $M^{o} \otimes_{R} N^{l} \otimes_{R} W$ using the left $R$-module $R \otimes 1^{o} W$. This is an $R^{\text {e-bimodule with }}$ actions

$$
\left(r \otimes t^{o}\right)\left(\sum_{i} m_{i}^{o} \otimes_{R} n_{i}^{l} \otimes_{R} w_{i}\right)\left(p \otimes q^{o}\right)=\sum_{i} r m_{i}^{o} \otimes_{R}\left(n_{i}\left(p \otimes q^{o}\right)\right)^{l} \otimes_{R} w_{i}\left(t \otimes 1^{o}\right),
$$

for every elements $\sum_{i} m_{i}^{o} \otimes_{R} n_{i}^{l} \otimes_{R} w_{i} \in M^{o} \otimes_{R} N^{l} \otimes_{R} W$ and $p, q, r, t \in R$.

Lastly, $M \times_{R} N \times_{R} W$ is defined to be the $R^{\mathrm{e}}$-invariant submodule with respect to the $R^{\mathrm{e}}$-biaction (17), that is,

$$
\begin{aligned}
& M \times_{R} N \times_{R} W=\left(M^{o} \otimes_{R} N^{l} \otimes_{R} W\right)^{R^{\mathrm{e}}}= \\
& \left\{\sum_{i} m_{i}^{o} \otimes_{R} n_{i}^{l} \otimes_{R} w_{i} \mid \sum_{i} r m_{i}^{o} \otimes_{R} n_{i}^{l} \otimes_{R} w\left(s \otimes 1^{o}\right)=\sum_{i} m_{i}^{o} \otimes_{R}\left(n_{i}\left(r \otimes s^{o}\right)\right)^{l} \otimes_{R} w, \text { for all } r, s \in R\right\} .
\end{aligned}
$$

The $\mathbb{k}$-module $M \times_{R} N \times_{R} W$ admits a structure of an $R^{\mathrm{e}}$-bimodule given by

$\left(r \otimes s^{o}\right)\left(\sum_{i} m_{i} \times_{R} n_{i} \times_{R} w_{i}\right)\left(p \otimes q^{o}\right)=\sum_{i}\left(\left(r \otimes 1^{o}\right) m_{i}\left(p \otimes 1^{o}\right)\right) \times_{R} n_{i} \times_{R}\left(\left(1 \otimes s^{o}\right) w_{i}\left(1 \otimes q^{o}\right)\right)$,

for every elements $\sum_{i} m_{i} \times_{R} n_{i} \times_{R} w_{i} \in M \times_{R} N \times_{R} W$ and $r, s, p, q \in R$.

The bi-functor $-\times_{R}-$ is not associative. However, the are natural $R^{\mathrm{e}}$-bilinear maps $\boldsymbol{\alpha}_{l}:\left(M \times_{R} N\right) \times_{R} W \longrightarrow M \times_{R} N \times_{R} W, \quad\left(\sum_{i}\left(\sum_{j} m_{i j} \times_{R} n_{i j}\right) \times_{R} w_{i} \longmapsto \sum_{i, j} m_{i j} \times_{R} n_{i j} \times_{R} w_{i}\right)$, $\boldsymbol{\alpha}_{r}: M \times_{R}\left(N \times_{R} W\right) \longrightarrow M \times_{R} N \times_{R} W, \quad\left(\sum_{i} m_{i} \times_{R}\left(\sum_{j} n_{i j} \times_{R} w_{i j}\right) \longmapsto \sum_{i, j} m_{i} \times_{R} n_{i j} \times_{R} w_{i j}\right)$.

The following lemma will be used in the sequel. 
Lemma 1.2.2. Let $N$ be an R-bimodule such that ${ }_{R} N$ is finitely generated and projective with left dual basis $\left\{\left(e_{j},{ }^{*} e_{j}\right)\right\}_{1 \leq j \leq m} \subset N \times^{*} N$. Consider the bimodule $R_{R^{e}} \mathcal{N}_{R^{\mathrm{e}}}=\left(N \otimes{ }^{*} N\right)^{\dagger}$. Then there is a well-defined map

$$
N \longrightarrow\left(\mathcal{N} \times_{R} \mathcal{N}\right) \times_{R} N, \quad\left(n \longmapsto \sum_{i, j}\left(\left(n \otimes{ }^{*} e_{j}\right)^{\dagger} \times_{R}\left(e_{j} \otimes{ }^{*} e_{i}\right)^{\dagger}\right) \times_{R} e_{i}\right) .
$$

Proof. Straightforward.

Another useful natural transformation of $R^{\mathrm{e}}$-bimodules is given as follows, see [10, p. 206]: For every $R^{\mathrm{e}}$-bimodules $M, M^{\prime}, N, N^{\prime}$, we have an $R^{\mathrm{e}}$-bilinear map:

$$
\begin{gathered}
\left(M \times_{R} M^{\prime}\right) \otimes_{R^{\mathrm{e}}}\left(N \times_{R} N^{\prime}\right) \stackrel{\tau}{\longrightarrow}\left(M \otimes_{R^{\mathrm{e}}} N\right) \times_{R}\left(M^{\prime} \otimes_{R^{\mathrm{e}}} N^{\prime}\right) \\
\left(\sum_{i} m_{i} \times_{R} m_{i}^{\prime}\right) \otimes_{R^{\mathrm{e}}}\left(\sum_{j} n_{j} \times_{R} n_{j}^{\prime}\right) \longmapsto \sum_{i, j}\left(m_{i} \otimes_{R^{\mathrm{e}}} n_{j}\right) \times_{R}\left(m_{i}^{\prime} \otimes_{R^{\mathrm{e}}} n_{j}^{\prime}\right) .
\end{gathered}
$$

In this way, given two $R^{\mathrm{e}}$-rings $S$ and $T$, then $S \times_{R} T$ is also an $R^{\mathrm{e}}$-ring.

It is clear that the $\mathbb{k}$-linear endomorphisms ring $\operatorname{End}_{\mathbb{k}}(R)$ is an $R^{\mathrm{e}}$-ring via the map $\varrho: R^{\mathrm{e}} \rightarrow \operatorname{End}_{\mathbb{k}}(R)$ which sends $p \otimes q^{o}$ to $[r \mapsto p r q]$. Given a pair of bimodules ${ }_{R^{o}} \mathbf{M}_{R^{o}}$ and ${ }_{R} \mathbf{N}_{R}$, there are two bilinear maps, see $[14, \S 2]$

$$
\begin{array}{cr}
\theta_{r}: \mathbf{M} \times_{R} \operatorname{End}_{\mathbb{k}}(R) \longrightarrow \mathbf{M}, & \theta_{l}: \operatorname{End}_{\mathbb{k}}(R) \times_{R} \mathbf{N} \longrightarrow \mathbf{N} \\
\sum_{i} m_{i} \times_{R} f_{i} \longmapsto \sum_{i} f_{i}(1)^{o} m_{i} & \sum_{j} g_{j} \times_{R} n_{j} \longmapsto
\end{array}
$$

If $M$ and $N$ are two $R^{\mathrm{e}}$-bimodules, then $\theta_{r}$ and $\theta_{l}$ are defined using the underlying bimodules $1 \otimes R^{o} M_{1 \otimes R^{o}}$ and ${ }_{R \otimes 1^{o}} N_{R \otimes 1^{o}}$, and both maps are $R^{\mathrm{e}}$-bilinear. Thus,

$$
\theta_{r}\left(\sum_{i} m_{i} \times_{R} f_{i}\right)=\left(1 \otimes f_{i}(1)^{o}\right) m_{i}, \text { and } \theta_{l}\left(\sum_{j} g_{j} \times_{R} n_{j}\right)=\left(g_{j}(1) \otimes 1^{o}\right) n_{j} .
$$

Recall from [14, $\S 4$, Definition 4.5] (see also [1] and [10]) the definition of $\times_{R^{-}}$-bialgebra. A $\times{ }_{R}$-coalgebra is an $R^{\mathrm{e}}$-bimodule $\mathrm{C}$ together with two $R^{\mathrm{e}}$-bilinear maps $\Delta: \mathrm{C} \rightarrow \mathrm{C} \times{ }_{R} \mathrm{C}$ (comultiplication) and $\varepsilon: \mathrm{C} \rightarrow \operatorname{End}_{\mathbb{k}}(R)$ (counit) such that the diagrams

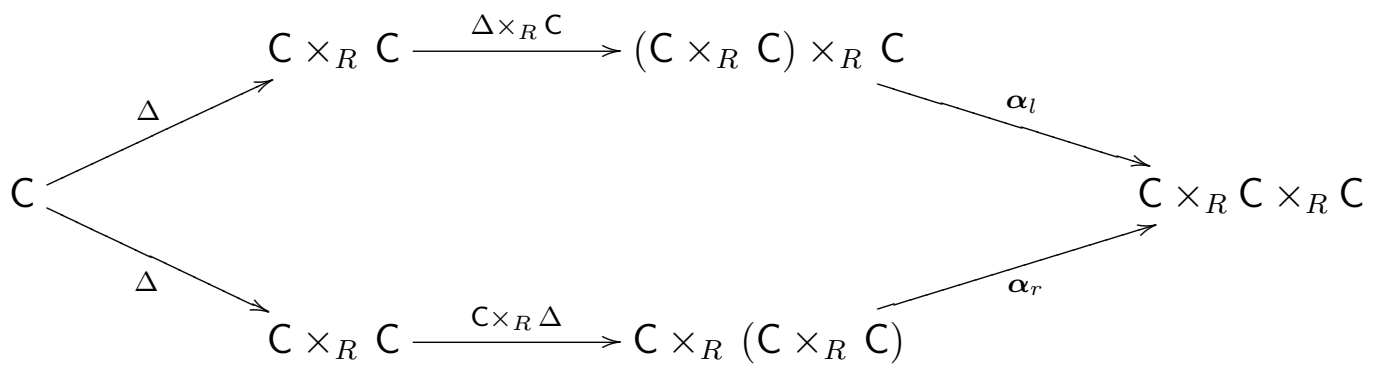




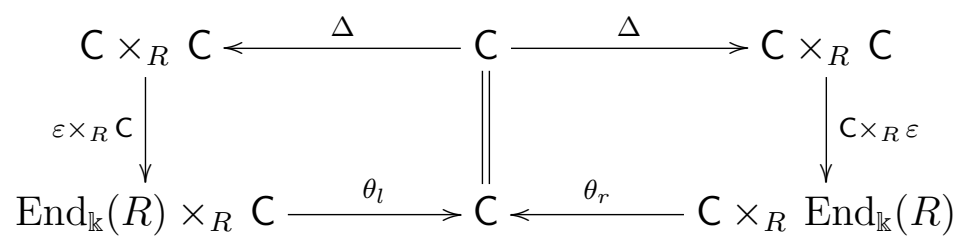

are commutative. $\mathrm{A} \times_{R^{-}}$-coalgebra $\mathrm{C}$ is said to be a $\times_{R^{-}}$bialgebra provided that comultiplication and counit are morphisms of $R^{\mathrm{e}}$-rings.

The underlying $R$-coring structure is given by

$$
\mathrm{C} \longrightarrow \mathrm{C} \times_{R} \mathrm{C} \stackrel{\Theta_{C, C}}{\longrightarrow} \mathrm{C}^{l} \otimes_{R} \mathrm{Cl}^{l}, \quad \mathrm{C} \stackrel{\varepsilon(-)\left(1_{R}\right)}{\longrightarrow} R,
$$

where $\Theta_{-,-}$is the natural transformation of (16).

1.3. The $\times_{R}$-bialgebra $\mathscr{L}_{m}(A)$. Let $A$ be an $R$-ring. Using the bifunctor of Subsection 1.2 , we get a functor $-\times_{R} A: R_{R} \operatorname{Mod}_{R} \rightarrow_{R} \operatorname{Mod}_{R}$. Now, for every pair of $R^{\mathrm{e}}$-bimodules $M$ and $N$, we have well-defined and $R$-bilinear maps:

$$
\begin{aligned}
& \left(M \times_{R} A\right) \otimes_{R}\left(N \times_{R} A\right) \stackrel{\Phi_{(M, N)}^{2}}{\longrightarrow}\left(M \otimes_{R^{\mathrm{e}}} N\right) \times_{R} A, \quad R \stackrel{\Phi^{0}}{\longrightarrow} R^{\mathrm{e}} \times_{R} A \\
& \left(m \times_{R} a\right) \otimes_{R}\left(n \times_{R} a^{\prime}\right) \longmapsto\left(m \otimes \otimes_{R^{\mathrm{e}}} n\right) \times_{R} a a^{\prime} \quad r \longmapsto \times_{R} 1_{A},
\end{aligned}
$$

where $\Phi_{(-,-)}^{2}$ is obviously a natural transformation. Thus we can state:

Lemma 1.3.3. Let $A$ be an R-ring. Then $-\times_{R} A:{ }_{R^{e}} \operatorname{Mod}_{R^{\mathrm{e}}} \rightarrow{ }_{R} \operatorname{Mod}_{R}$ is a monoidal functor with structure maps $\Phi_{(-,-)}^{2}$ and $\Phi^{0}$ of Eq. (19).

Proof. These are routine verifications.

¿From now on, we assume that our $R$-ring $A$ is finitely generated and projective as left $R$-module. We fix a left dual basis $\left\{\left(e_{j},{ }^{*} e_{j}\right)\right\}_{1 \leq j \leq n} \subset A \times{ }^{*} A$. By Lemma 1.1.1,

$$
\mathscr{R}:=-\times_{R} A:{ }_{R} \operatorname{Mod}_{R}{ }^{\mathrm{e}} \longrightarrow{ }_{R} \operatorname{Mod}_{R}
$$

is a right adjoint to the functor

$$
\mathscr{L}:=\left(-\otimes{ }^{*} A\right)^{\dagger}:{ }_{R} \operatorname{Mod}_{R} \longrightarrow{ }_{R^{\mathrm{e}}} \operatorname{Mod}_{R^{\mathrm{e}}} .
$$

The unit and counit of this adjunction are explicitly given as follows. For any $R$-bimodule $X$ and any $R^{\mathrm{e}}$-bimodule $U$, the unit at the object $X$ is given by

$$
\begin{gathered}
X \stackrel{\eta_{X}}{\longrightarrow} \mathscr{R} \mathscr{L}(X)=\left(X \otimes{ }^{*} A\right)^{\dagger} \times_{R} A, \\
x \longmapsto \sum_{j}\left(x \otimes{ }^{*} e_{j}\right)^{\dagger} \times_{R} e_{j},
\end{gathered}
$$

while the counit at $U$ is given by

$$
\begin{aligned}
\mathscr{L} \mathscr{R}(U) & =\left(\left(U \times_{R} A\right) \otimes{ }^{*} A\right) \dagger \stackrel{\xi_{U}}{\longrightarrow} U \\
\left(\left(u \times_{R} a\right) \otimes \varphi\right)^{\dagger} \longmapsto & \longrightarrow\left(1 \otimes \varphi(a)^{o}\right) u .
\end{aligned}
$$


The functor $\mathscr{L}:{ }_{R} \operatorname{Mod}_{R} \rightarrow{ }_{R} \operatorname{Mod}_{R}$ is a comonoidal functor whose structures maps, using (20) and (21), are given by

$$
\begin{aligned}
& \left(\left(X \otimes_{R} Y\right) \otimes{ }^{*} A\right)^{\dagger} \stackrel{\Psi_{(X, Y)}^{2}}{\longrightarrow}\left(X \otimes *^{*} A\right)^{\dagger} \otimes_{R^{\mathrm{e}}}\left(Y \otimes \otimes^{*} A\right)^{\dagger}, \quad\left(R \otimes *^{*} A\right)^{\dagger} \stackrel{\Psi^{0}}{\longrightarrow} R^{\mathrm{e}} \\
& \left(\left(x \otimes_{R} y\right) \otimes \varphi\right)^{\dagger} \longmapsto \\
& \sum_{i}\left(x \otimes e_{j} \varphi\right)^{\dagger} \otimes_{R^{\mathrm{e}}}\left(y \otimes \otimes^{*} e_{j}\right)^{\dagger} \quad(r \otimes \varphi)^{\dagger} \longmapsto
\end{aligned}
$$

for every pair of $R$-bimodules $X$ and $Y$.

The following is a classical result in the theory of monoidal categories (see for instance [15, Remark 1.5]). We will freely use it in the construction performed in the sequel.

TheOREm 1.3.4. Let $\left(\mathcal{B}, \otimes_{\mathcal{B}}, \mathbf{1}_{\mathcal{B}}\right)$ and $\left(\mathcal{C}, \otimes_{\mathcal{C}}, \mathbf{1}_{\mathcal{C}}\right)$ be monoidal categories. Let $\mathscr{L} \dashv \mathscr{R}$ be an adjunction where $\mathscr{R}: \mathcal{C} \rightarrow \mathcal{B}$ is a monoidal functor with structure morphisms $\Phi_{(-,-)}^{2}$ and $\Phi^{0}$. Then $\mathscr{R}$ induces a functor $\mathscr{R}_{m}: \mathcal{C}_{m} \rightarrow \mathcal{B}_{m}$ between the associated categories of monoids.

Assume that $\mathcal{C}$ has inductive limits and that the tensor product preserves them. Then $\mathscr{R}_{m}$ has a left adjoint $\mathscr{L}_{m}: \mathcal{B}_{m} \rightarrow \mathcal{C}_{m}$.

By Theorem 1.3.4 and Lemma 1.3.3, the adjunction $\mathscr{L} \dashv \mathscr{R}$ of (20)-(21) restricts to the categories of ring extension. That is, we have an adjunction

$$
\mathscr{L}_{m}: R \text {-Rings } \longleftrightarrow R^{\mathrm{e}} \text {-Rings : } \mathscr{R}_{m} \text {. }
$$

For a given $R$-ring $C$, i.e. a $\mathbb{k}$-algebra map $R \rightarrow C$, the $R^{\mathrm{e}}$-ring $\mathscr{L}_{m}(C)$ is defined, by the quotient algebra

$$
\mathscr{L}_{m}(C)=\mathscr{T}_{R^{\mathrm{e}}}(\mathscr{L}(C)) / \mathscr{I}_{\mathscr{L}(C)}
$$

where $\mathscr{T}_{R^{\mathrm{e}}}(\mathscr{L}(C))=\bigoplus_{n \in \mathbb{N}} \mathscr{L}(C)^{\otimes_{R^{\mathrm{e}}}}$ is the tensor algebra of the $R^{\mathrm{e}}$-bimodule $\mathscr{L}(C)=$ $\left(C \otimes{ }^{*} A\right)^{\dagger}$ and where $\mathscr{I}_{\mathscr{L}(C)}$ is the two-sided ideal generated by the set

$$
\left\{\sum_{i}\left(\left(c \otimes e_{i} \varphi\right)^{\dagger} \otimes R^{\mathrm{e}}\left(c^{\prime} \otimes{ }^{*} e_{i}\right)^{\dagger}\right)-\left(c c^{\prime} \otimes \varphi\right)^{\dagger} ; 1_{R} \otimes \varphi\left(1_{A}\right)^{o}-\left(1_{C} \otimes \varphi\right)^{\dagger}\right\}_{c, c^{\prime} \in C, \varphi \in{ }^{*} A}
$$

We denote by $\pi_{C}: \mathscr{T}_{R^{\mathrm{e}}}(\mathscr{L}(C)) \rightarrow \mathscr{L}_{m}(C)$ the canonical projection. From now on, given a homogeneous element $(c \otimes \varphi)^{\dagger} \in \mathscr{T}_{R^{\mathrm{e}}}(C)$ of degree one, we denote by $\pi_{C}(c \otimes \varphi)$ its image in the $R^{\mathrm{e}}$-ring $\mathscr{L}_{m}(C)$. Thus, throughout this section we will drop the symbol dag in the upper indices, and consider $C \otimes{ }^{*} A$ as an $R^{\mathrm{e}}$-bimodule with its dag biaction, see (5).

The unit and counit of the adjunction $(22)$, can be written as follows:

$$
\begin{aligned}
C \longrightarrow \eta_{C}^{m} \longrightarrow & \mathscr{R}_{m} \mathscr{L}_{m}(C)=\mathscr{L}_{m}(C) \times_{R} A, \\
c \longmapsto & \sum_{j} \pi_{C}\left(c \otimes{ }^{*} e_{j}\right) \times_{R} e_{j}
\end{aligned}
$$




$$
\begin{aligned}
\mathscr{L}_{m} \mathscr{R}_{m}(B) & =\left(\left(B \times_{R} A\right) \otimes{ }^{*} A\right) \longrightarrow B \\
\pi_{\mathscr{L}_{m}(B)}\left(\left(b \times_{R} a\right) \otimes \varphi\right) & \longmapsto
\end{aligned}
$$

for every $R$-ring $C$ and $R^{\mathrm{e}}$-ring $B$. Notice that $\xi_{B}^{m}$ is defined by the universal property of the tensor algebra.

Next, we proceed to show that $\mathscr{L}_{m}(A)$ is an $\times_{R}$-bialgebra. The structure of an $R^{\mathrm{e}}$-ring, is given by the following composition of algebra maps

$$
R^{\mathrm{e}} \stackrel{\iota_{0}}{\longrightarrow} \mathscr{T}_{R^{\mathrm{e}}}(\mathscr{L}(A)) \stackrel{\pi_{A}}{\longrightarrow} \mathscr{L}_{m}(A),
$$

where $\iota_{n}$ denotes the canonical $R^{\mathrm{e}}$-bilinear injection in degree $n \geq 0$.

Lemma 1.3.5. Let $A$ be an $R$-ring which is finitely generated and projective as left $R$-module with dual basis $\left\{\left({ }^{*} e_{i}, e_{i}\right)\right\}_{i}$. The following maps

$$
\begin{gathered}
\delta: A \longrightarrow\left(\mathscr{L}_{m}(A) \times_{R} \mathscr{L}_{m}(A)\right) \times_{R} A, \quad\left(a \longmapsto \sum_{j, i}\left(\pi_{A}\left(a \otimes * e_{j}\right) \times_{R} \pi_{A}\left(e_{j} \otimes{ }^{*} e_{i}\right)\right) \times_{R} e_{i}\right) \\
\omega: A \longrightarrow \operatorname{End}_{\mathbb{k}}(R) \times_{R} A, \quad\left(a \longmapsto \sum_{j}{ }^{*} e_{j}(a \bullet) \times_{R} e_{j}\right), \text { where }\left[{ }^{*} e_{j}(a \bullet): r \mapsto{ }^{*} e_{j}(a r)\right]
\end{gathered}
$$

are morphisms of $R$-rings.

Proof. We only prove that $\delta$ is a morphism of $R$-rings. Similar arguments are used to show that $\omega$ is also a morphism of $R$-rings. The map $\delta$ is in fact the composition of the following two maps

$$
\delta: A \longrightarrow\left(\mathscr{L}(A) \times_{R} \mathscr{L}(A)\right) \times_{R} A \stackrel{\left(\pi_{A} \mathrm{o \iota}_{1} \times_{R} \pi_{A} \circ_{1}\right) \times_{R} A}{\longrightarrow}\left(\mathscr{L}_{m}(A) \times_{R} \mathscr{L}_{m}(A)\right) \times_{R} A,
$$

where the first one is defined via Lemma 1.2.2. Thus $\delta$ is a well-defined map. Now, let us show that $\delta$ is a morphism of $R$-rings. The unit is preserved by $\delta$, since we have

$$
\begin{aligned}
\delta\left(1_{A}\right) & =\sum_{j, i}\left(\pi_{A}\left(1_{A} \otimes{ }^{*} e_{j}\right) \times_{R} \pi_{A}\left(e_{j} \otimes{ }^{*} e_{i}\right)\right) \times_{R} e_{i}, \quad\left(\pi_{A}\left(1_{A} \otimes{ }^{*} e_{j}\right)=\pi_{A}\left(1_{R} \otimes{ }^{*} e_{j}\left(1_{A}\right)^{o}\right)\right) \\
& =\sum_{j, i}\left(\pi_{A}\left(1_{R} \otimes{ }^{*} e_{j}\left(1_{A}\right)^{o}\right) \times_{R} \pi_{A}\left(e_{j} \otimes^{*} e_{i}\right)\right) \times_{R} e_{i} \\
& =\sum_{j, i}\left(\left(\left(1 \otimes{ }^{*} e_{j}\left(1_{A}\right)^{o}\right) \cdot \pi_{A}\left(1_{R^{\mathrm{e}}}\right)\right) \times_{R} \pi_{A}\left(e_{j} \otimes{ }^{*} e_{i}\right)\right) \times_{R} e_{i} \\
& =\sum_{j, i}\left(\pi_{A}\left(1_{R^{\mathrm{e}}}\right) \times_{R}\left({ }^{*} e_{j}\left(1_{A}\right) \cdot \pi_{A}\left(e_{j} \otimes^{*} e_{i}\right)\right)\right) \times_{R} e_{i} \\
& =\sum_{j, i}\left(\pi_{A}\left(1_{R^{\mathrm{e}}}\right) \times_{R} \pi_{A}\left({ }^{*} e_{j}\left(1_{A}\right) e_{j} \otimes{ }^{*} e_{i}\right)\right) \times_{R} e_{i} \\
& =\sum_{i}\left(\pi_{A}\left(1_{R^{\mathrm{e}}}\right) \times_{R} \pi_{A}\left(1_{A} \otimes{ }^{*} e_{i}\right)\right) \times_{R} e_{i}
\end{aligned}
$$




$$
\begin{aligned}
& =\sum_{i}\left[\left(\pi_{A}\left(1_{R^{\mathrm{e}}}\right) \times_{R} \pi_{A}\left(1_{R^{\mathrm{e}}}\right)\right)\left(1 \otimes{ }^{*} e_{i}\left(1_{A}\right)^{o}\right)\right] \times_{R} e_{i} \\
& \stackrel{(10)}{=} \sum_{i}\left(\pi_{A}\left(1_{R^{\mathrm{e}}}\right) \times_{R} \pi_{A}\left(1_{R^{\mathrm{e}}}\right)\right) \times_{R} e_{i}^{*} e_{i}\left(1_{A}\right) \\
& =\left(\pi_{A}\left(1_{R^{\mathrm{e}}}\right) \times_{R} \pi_{A}\left(1_{R^{\mathrm{e}}}\right)\right) \times_{R} 1_{A} .
\end{aligned}
$$

For any $a, a^{\prime} \in A$, we have

$$
\begin{aligned}
& \delta\left(a a^{\prime}\right)= \\
& =\quad \sum_{j, i}\left[\pi_{A}\left(a a^{\prime} \otimes{ }^{*} e_{j}\right) \times_{R} \pi_{A}\left(e_{j} \otimes{ }^{*} e_{i}\right)\right] \times_{R} e_{i} \\
& =\quad \sum_{j, i, k}\left[\pi_{A}\left(\left(a \otimes e_{k}^{*} e_{j}\right) \otimes_{R^{\mathrm{e}}}\left(a^{\prime} \otimes{ }^{*} e_{k}\right)\right) \times_{R} \pi_{A}\left(e_{j} \otimes{ }^{*} e_{i}\right)\right] \times_{R} e_{i} \\
& =\sum_{j, i, k, l}\left[\pi_{A}\left(\left(a \otimes{ }^{*} e_{l}^{*} e_{j}\left(e_{l} e_{k}\right)\right) \otimes_{R^{\mathrm{e}}}\left(a^{\prime} \otimes{ }^{*} e_{k}\right)\right) \times_{R} \pi_{A}\left(e_{j} \otimes{ }^{*} e_{i}\right)\right] \times_{R} e_{i} \\
& =\sum_{j, i, k, l}\left[\left(\pi_{A}\left(\left(a \otimes^{*} e_{l}\right) \otimes_{R^{\mathrm{e}}}\left(a^{\prime} \otimes^{*} e_{k}\right)\right){ }^{*} e_{j}\left(e_{l} e_{k}\right)\right) \times_{R} \pi_{A}\left(e_{j} \otimes{ }^{*} e_{i}\right)\right] \times_{R} e_{i} \\
& =\sum_{j, i, k, l}\left[\pi_{A}\left(\left(a \otimes{ }^{*} e_{l}\right) \otimes_{R^{\mathrm{e}}}\left(a^{\prime} \otimes{ }^{*} e_{k}\right)\right) \times_{R}\left(\left({ }^{*} e_{j}\left(e_{l} e_{k}\right) \otimes 1_{R}^{o}\right) \pi_{A}\left(e_{j} \otimes{ }^{*} e_{i}\right)\right)\right] \times_{R} e_{i} \\
& =\sum_{j, i, k, l}\left[\pi_{A}\left(\left(a \otimes{ }^{*} e_{l}\right) \otimes_{R^{e}}\left(a^{\prime} \otimes{ }^{*} e_{k}\right)\right) \times_{R} \pi_{A}\left({ }^{*} e_{j}\left(e_{l} e_{k}\right) e_{j} \otimes{ }^{*} e_{i}\right)\right] \times_{R} e_{i} \\
& =\sum_{i, k, l}\left[\pi_{A}\left(\left(a \otimes{ }^{*} e_{l}\right) \otimes_{R^{e}}\left(a^{\prime} \otimes{ }^{*} e_{k}\right)\right) \times_{R} \pi_{A}\left(e_{l} e_{k} \otimes{ }^{*} e_{i}\right)\right] \times_{R} e_{i} \\
& =\sum_{i, k, l, m}\left[\pi_{A}\left(\left(a \otimes^{*} e_{l}\right) \otimes_{R^{\mathrm{e}}}\left(a^{\prime} \otimes^{*} e_{k}\right)\right) \times_{R} \pi_{A}\left(\left(e_{l} \otimes e_{m}{ }^{*} e_{i}\right) \otimes_{R^{\mathrm{e}}}\left(e_{k} \otimes^{*} e_{m}\right)\right)\right] \times_{R} e_{i} \\
& =\sum_{i, k, l, m, n}\left[\pi_{A}\left(\left(a \otimes^{*} e_{l}\right) \otimes_{R^{\mathrm{e}}}\left(a^{\prime} \otimes^{*} e_{k}\right)\right) \times_{R} \pi_{A}\left(\left(e_{l} \otimes^{*} e_{n}{ }^{*} e_{i}\left(e_{n} e_{m}\right)\right) \otimes_{R^{\mathrm{e}}}\left(e_{k} \otimes^{*} e_{m}\right)\right)\right] \times_{R} e_{i} \\
& =\sum_{i, k, l, m, n}\left[\pi_{A}\left(\left(a \otimes{ }^{*} e_{l}\right) \otimes_{R^{\mathrm{e}}}\left(a^{\prime} \otimes{ }^{*} e_{k}\right)\right) \times_{R}\left(\left(1 \otimes^{*} e_{i}\left(e_{n} e_{m}\right)^{o}\right) \pi_{A}\left(\left(e_{l} \otimes^{*} e_{n}\right) \otimes_{R^{\mathrm{e}}}\left(e_{k} \otimes^{*} e_{m}\right)\right)\right)\right] \times_{R} e_{i} \\
& \stackrel{(15)}{=} \sum_{i, k, l, m, n}\left[\left(1 \otimes^{*} e_{i}\left(e_{n} e_{m}\right)^{o}\right)\left[\pi_{A}\left(\left(a \otimes \otimes^{*} e_{l}\right) \otimes_{R^{\mathrm{e}}}\left(a^{\prime} \otimes^{*} e_{k}\right)\right) \times_{R} \pi_{A}\left(\left(e_{l} \otimes^{*} e_{n}\right) \otimes_{R^{\mathrm{e}}}\left(e_{k} \otimes^{*} e_{m}\right)\right)\right]\right] \times_{R} e_{i} \\
& =\sum_{i, k, l, m, n}\left[\pi_{A}\left(\left(a \otimes^{*} e_{l}\right) \otimes_{R^{\mathrm{e}}}\left(a^{\prime} \otimes^{*} e_{k}\right)\right) \times_{R} \pi_{A}\left(\left(e_{l} \otimes^{*} e_{n}\right) \otimes_{R^{\mathrm{e}}}\left(e_{k} \otimes{ }^{*} e_{m}\right)\right)\right] \times_{R}{ }^{*} e_{i}\left(e_{n} e_{m}\right) e_{i} \\
& =\sum_{k, l, m, n}\left[\pi_{A}\left(\left(a \otimes{ }^{*} e_{l}\right) \otimes_{R^{\mathrm{e}}}\left(a^{\prime} \otimes{ }^{*} e_{k}\right)\right) \times_{R} \pi_{A}\left(\left(e_{l} \otimes^{*} e_{n}\right) \otimes_{R^{\mathrm{e}}}\left(e_{k} \otimes{ }^{*} e_{m}\right)\right)\right] \times_{R} e_{n} e_{m} \\
& =\sum_{k, l, m, n}\left[\left(\pi_{A}\left(a \otimes{ }^{*} e_{l}\right) \times_{R} \pi_{A}\left(e_{l} \otimes{ }^{*} e_{n}\right)\right)\left(\pi_{A}\left(a^{\prime} \otimes{ }^{*} e_{k}\right) \times_{R} \pi_{A}\left(e_{k} \otimes{ }^{*} e_{m}\right)\right)\right] \times_{R} e_{n} e_{m} \\
& =\left[\sum_{l, n}\left(\pi_{A}\left(a \otimes{ }^{*} e_{l}\right) \times_{R} \pi_{A}\left(e_{l} \otimes{ }^{*} e_{n}\right)\right) \times_{R} e_{n}\right]\left[\sum_{k, m}\left(\pi_{A}\left(a^{\prime} \otimes{ }^{*} e_{k}\right) \times_{R} \pi_{A}\left(e_{k} \otimes{ }^{*} e_{m}\right)\right) \times_{R} e_{m}\right] \\
& =\delta(a) \delta\left(a^{\prime}\right),
\end{aligned}
$$


and hence $\delta\left(a a^{\prime}\right)=\delta(a) \delta\left(a^{\prime}\right)$, which shows that $\delta$ is multiplicative. A similar computation shows that $\delta$ satisfies

$$
\delta\left(r 1_{A}\right)=\left(\pi_{A}\left(r 1_{R^{\mathrm{e}}}\right) \times_{R} \pi_{A}\left(1_{R^{\mathrm{e}}}\right)\right) \times_{R} 1_{A}=r 1_{\mathscr{L}_{m}(A) \times_{R} \mathscr{L}_{m}(A)} \times_{R} 1_{A}, \quad \text { for every } r \in R
$$

which means that the diagram

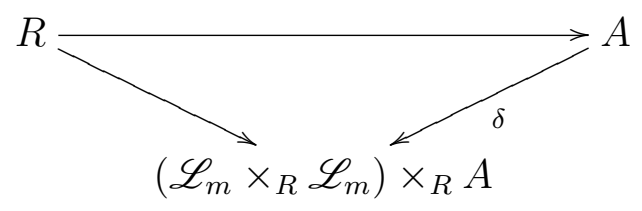

is commutative, and this finishes the proof.

Part of the following proposition was first observed by D. Tambara in [15, Remark 1.7] with no proof. Note that, from a categorical point of view, one can expect that this is rather a trivial result. However, this is far from being a direct or immediate verification, since the handled categories as we have seen have a very complicated monoidal structure which is due to the fact that we are dealing with multi-modules over $R$ rather than fixed bimodules and that the product $\times_{R}$ is not associative.

Proposition 1.3.6. Let $A$ be an $R$-ring which is finitely generated and projective as a left $R$-module with dual basis $\left\{\left({ }^{*} e_{i}, e_{i}\right)\right\}_{i}$. Then $\mathscr{L}_{m}(A)$ is a $\times_{R}$-bialgebra with structure maps

$$
\begin{array}{ccc}
\mathscr{L}_{m}(A) \stackrel{\Delta}{\longrightarrow} \mathscr{L}_{m}(A) \times_{R} \mathscr{L}_{m}(A), & \mathscr{L}_{m}(A) \stackrel{\varepsilon}{\longrightarrow} \operatorname{End}_{\mathbb{k}}(R) \\
\pi_{A}(a \otimes \varphi) \longmapsto & \pi_{A}(a \otimes \varphi) \longmapsto
\end{array}
$$

Proof. Both $\Delta$ and $\varepsilon$ are defined via the adjunction $\mathscr{L}_{m} \dashv \mathscr{R}_{m}$ of Eq (25)-(26). In fact, we have

$$
\Delta=\xi_{\mathscr{L}_{m}(A) \times_{R} \mathscr{L}_{m}(A)}^{m} \circ \mathscr{L}_{m}(\delta)
$$

where $\delta$ is the morphism of $R$-rings defined in Lemma 1.3.5, and $\xi_{-}^{m}$ is the counit of the adjunction $\mathscr{L}_{m} \dashv \mathscr{R}_{m}$. Therefore, it is immediate that $\Delta$ is a morphism of $R^{\mathrm{e}}$-rings. To 
show that $\Delta$ is coassociative it suffices to check that the following diagram is commutative

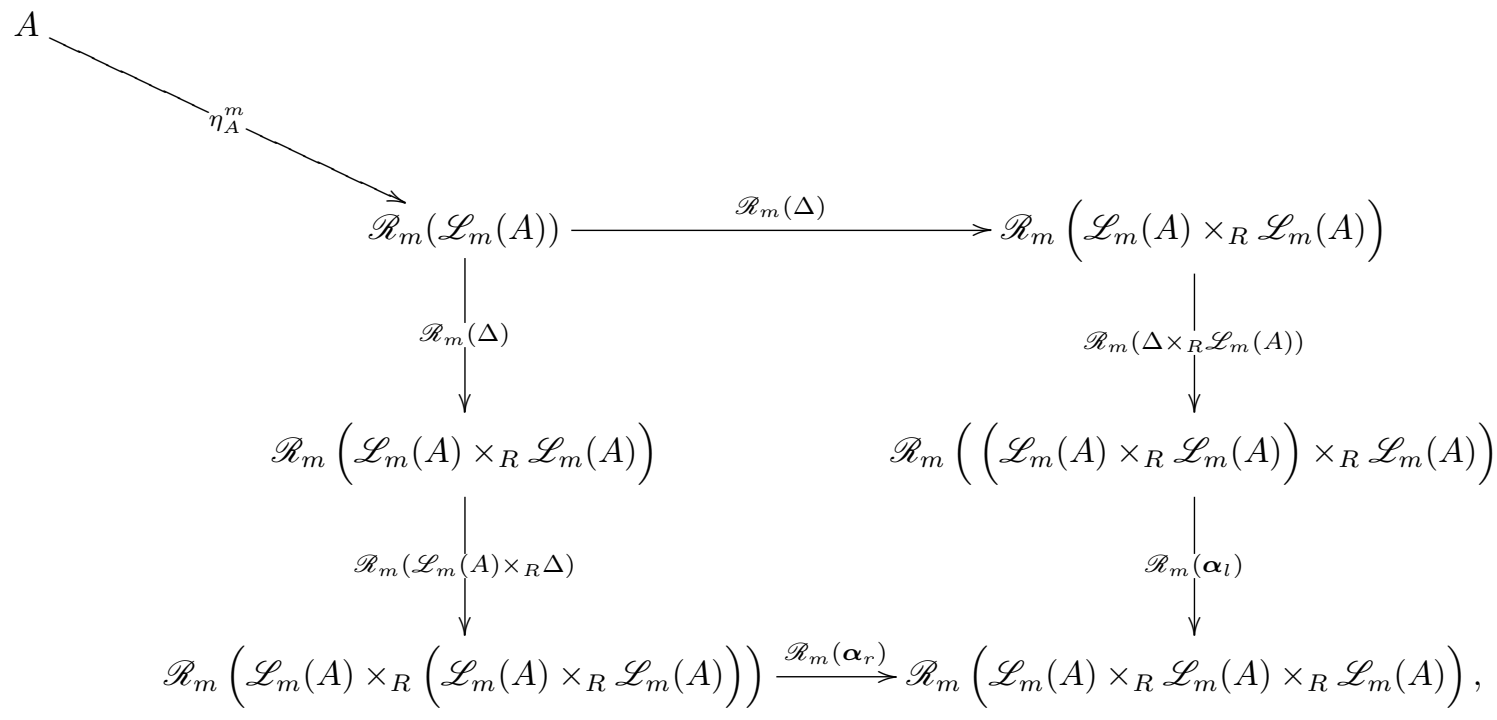

and this follows from routine computations.

We also have $\varepsilon=\xi_{\operatorname{End}_{\mathrm{k}}(R)}^{m} \circ \mathscr{L}_{m}(\omega)$, where $\omega: A \rightarrow \operatorname{End}_{\mathbb{k}}(R) \times_{R} A$ is the morphism of $R$-rings defined in Lemma 1.3.5. Hence $\varepsilon$ is clearly a morphism of $R^{\mathrm{e}}$-rings. Furthermore, it satisfies the following equality

$$
\theta_{l} \circ\left(\varepsilon \times_{R} \mathscr{L}_{m}(A)\right) \circ \Delta=\mathscr{L}_{m}(A)=\theta_{r} \circ\left(\mathscr{L}_{m}(A) \times_{R} \varepsilon\right) \circ \Delta
$$

which is the counitarity property.

Next we provide the relation between the $R$-ring structure of $A$ and the $\times_{R}$-bialgebra structure of $\mathscr{L}_{m}(A)$.

Corollary 1.3.7. Let $A$ be an $R$-ring such that ${ }_{R} A$ is finitely generated and projective and $\mathscr{L}_{m}(A)$ the associated $\times_{R}$-bialgebra defined in Proposition 1.3.6. Then $A$ is a left $\times_{R^{-}}$ $\mathscr{L}_{m}(A)$-comodule $R$-ring, that is, $A$ admits a left $\times_{R}-\mathscr{L}_{m}(A)$-coaction $\lambda_{A}: A \rightarrow \mathscr{L}_{m}(A) \times_{R}$ $A$ which is also a morphism of $R$-rings.

Proof. The unit of the adjunction given in $(22)$ at $A$

$$
\eta_{A}^{m}: A \longrightarrow \mathscr{L}_{m}(A) \times_{R} A, \quad\left(a \longmapsto \sum_{i} \pi_{A}\left(a \otimes{ }^{*} e_{i}\right) \times_{R} e_{i}\right)
$$


is by definition a morphism of $R$-rings. Let us check that it is a left $\mathscr{L}_{m}(A)$-coaction. It remains to show that the following diagrams are commutative

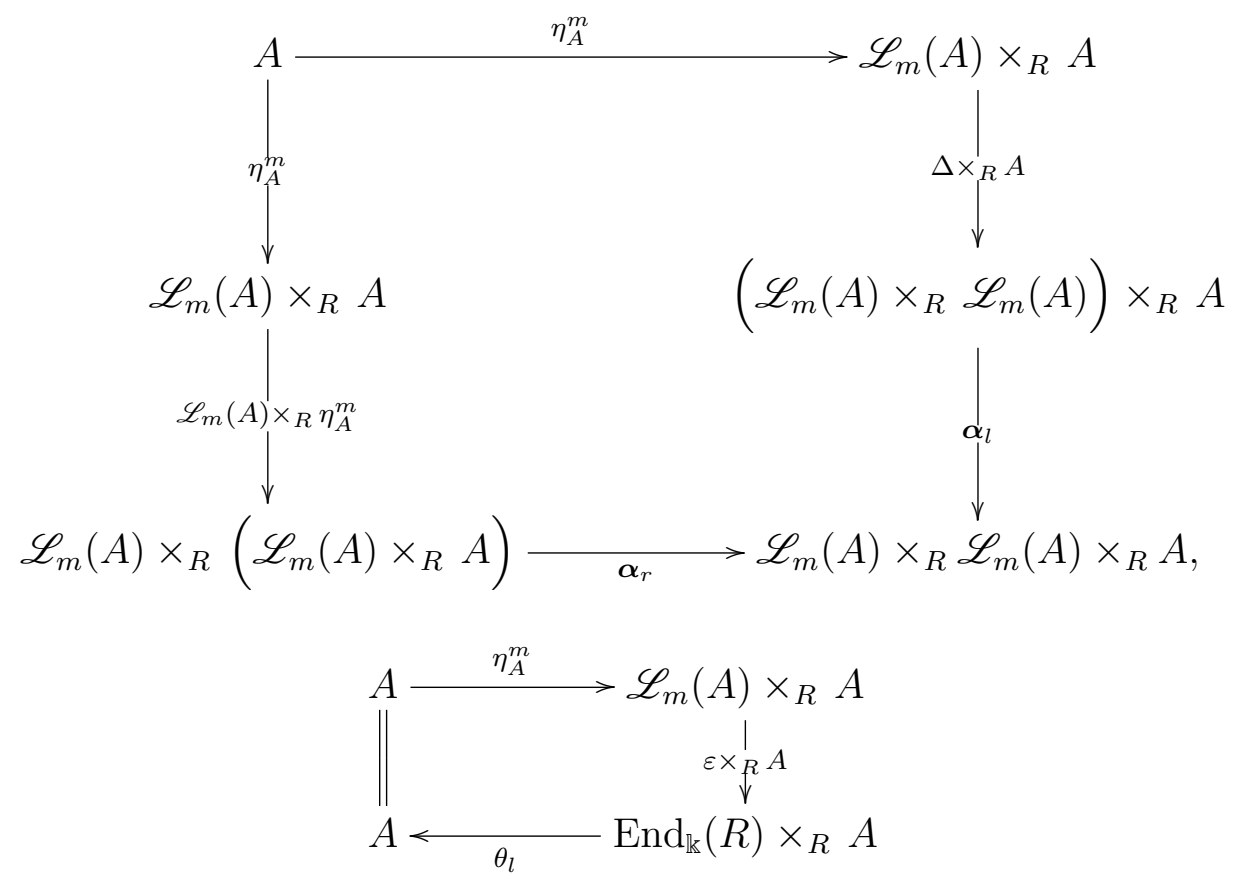

For every element $a \in A$, we have

$$
\begin{aligned}
\boldsymbol{\alpha}_{l} \circ\left(\Delta \times_{R} A\right) \circ \eta_{A}^{m}(a) & =\sum_{i, j} \boldsymbol{\alpha}_{l}\left(\left(\pi_{A}\left(a \otimes^{*} e_{i}\right) \times_{R} \pi_{A}\left(e_{i} \otimes^{*} e_{j}\right)\right) \times_{R} e_{j}\right) \\
& =\sum_{i, j} \pi_{A}\left(a \otimes^{*} e_{i}\right) \times_{R} \pi_{A}\left(e_{i} \otimes^{*} e_{j}\right) \times_{R} e_{j} \\
& =\sum_{i, j} \boldsymbol{\alpha}_{r}\left(\pi_{A}\left(a \otimes^{*} e_{j}\right) \times_{R}\left(\pi_{A}\left(e_{j} \otimes^{*} e_{i}\right) \times_{R} e_{i}\right)\right) \\
& =\boldsymbol{\alpha}_{r} \circ\left(\mathscr{L}_{m}(A) \times_{R} \eta_{A}^{m}\right) \circ\left(\sum_{i} \pi_{A}\left(a \otimes^{*} e_{i}\right) \times_{R} e_{i}\right) \\
& =\boldsymbol{\alpha}_{r} \circ\left(\mathscr{L}_{m}(A) \times_{R} \eta_{A}^{m}\right) \circ \eta_{A}^{m}(a),
\end{aligned}
$$

and also we have

$$
\begin{aligned}
\theta_{l} \circ\left(\varepsilon \times_{R} A\right) \circ \eta_{A}^{m}(a) & =\theta_{l} \circ\left(\varepsilon \times_{R} A\right)\left(\sum_{i} \pi_{A}\left(a \otimes^{*} e_{i}\right) \times_{R} e_{i}\right) \\
& =\sum_{i} \theta_{l}\left({ }^{*} e_{i}(a \bullet) \times_{R} e_{i}\right) \\
& =\sum_{i}^{*} e_{i}(a) e_{i}=a .
\end{aligned}
$$


This proves the commutativity of the above diagrams and establishes the corollary.

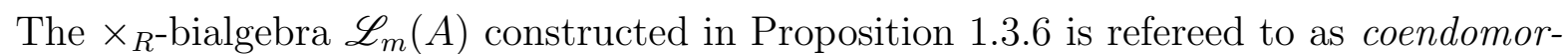
phism left $R$-bialgebroid since, by [1, Theorem 3.1], $\mathscr{L}_{m}(A)$ is in fact a (left) bialgebroid whose structure of $R^{\mathrm{e}}$-ring is the map

$$
\pi_{A} \circ \iota_{0}: R^{\mathrm{e}} \longrightarrow \mathscr{L}_{m}(A),
$$

and its structure of $R$-coring is given as follows. The underlying $R$-bimodule is $\mathscr{L}_{m}(A)^{l}=$ $R^{\mathrm{e}} \mathscr{L}_{m}(A)$, the comultiplication and counit are given by

$$
\begin{aligned}
\Delta: \mathscr{L}_{m}(A)^{l} \longrightarrow \mathscr{L}_{m}(A)^{l} \otimes_{R} \mathscr{L}_{m}(A)^{l}, & \\
& \left(\pi_{A}(a \otimes \varphi) \longmapsto \sum_{i} \pi_{A}\left(a \otimes{ }^{*} e_{i}\right) \otimes_{R} \pi_{A}\left(e_{i} \otimes \varphi\right)\right), \\
\varepsilon: \mathscr{L}_{m}(A)^{l} \longrightarrow & R, \quad\left(\pi_{A}(a \otimes \varphi) \longmapsto \varphi(a)\right) .
\end{aligned}
$$

REMARK 1.3.8. As one can realize the coendomorphism right bialgebroids are constructed by taking a $\mathbb{k}$-algebra extension $R \rightarrow A$ where $A_{R}$ is finitely generated and projective right module.

On the other hand, one may wish to have structures of left Hopf algebroids that can be extracted from coendomorphism left bialgebroids, as was done for some localizations of coendomorphisms bialgebras which give rise to new Hopf algebras. Perhaps in this general case one can use also the theory of noncommutative localization at some multiplicative set of grouplike elements. However, we believe that this is not an easy task which deserves a separate project.

\section{EXAMPLES OF COENDOMORPHISM BIALGEBROIDS.}

In this section we give some concrete examples of coendomorphism bialgebroids, by specifying their generators and relations.

EXAMPLE 2.0.1 (Quaternion coendomorphism bialgebra). Assume that $R=\mathbb{k}$ is a field with characteristic not equal to 2 . Let $A$ be the Hamilton quaternion $\mathbb{k}$-algebra associated to the pair $(-1,-1)$. That is, $A=\mathbb{k} \oplus \mathbb{k} \mathfrak{i} \oplus \mathbb{k} \mathfrak{j} \oplus \mathbb{k} \mathfrak{i j}$ with relation $\mathfrak{i}^{2}=-1=\mathfrak{j}^{2}$ and $\mathfrak{i j}=-\mathfrak{j i}$. Then one can prove, using Proposition 1.3.6, that $\mathscr{L}_{m}(A)$ is a $\mathbb{k}$-bialgebra, which is generated as a $\mathbb{k}$-algebra by elements $\left\{x_{k}, y_{k}, z_{k}, u_{k}\right\}_{1 \leq k \leq 3}$ subject to the relations

$$
\begin{aligned}
1+x_{k}^{2} & =y_{k}^{2}+z_{k}^{2}+u_{k}^{2}, \quad \text { for all } k=1,2,3, \\
x_{1} x_{2}+x_{2} x_{1} & =y_{2} y_{1}+y_{1} y_{2}+u_{2} u_{1}+u_{1} u_{2}+z_{2} z_{1}+z_{1} z_{2},
\end{aligned}
$$

$x_{1} y_{1}=-y_{1} x_{1}+z_{1} u_{1}-u_{1} z_{1}$,

$$
\begin{aligned}
z_{1} y_{1} & =y_{1} z_{1}-x_{1} u_{1}-u_{1} x_{1} \\
x_{3} & =x_{1} x_{2}-y_{1} y_{2}-z_{1} z_{2}-u_{1} u_{2} \\
y_{3} & =x_{1} y_{2}+y_{1} x_{2}-z_{1} u_{2}+u_{1} z_{2}
\end{aligned}
$$$$
u_{1} y_{1}=y_{1} u_{1}+z_{1} x_{1}+x_{1} z_{1} \text {, }
$$ 


$$
\begin{array}{rlrl}
z_{3} & =x_{1} z_{2}+y_{1} u_{2}+z_{1} x_{2}-u_{1} y_{2}, & u_{2} y_{1} & =x_{2} z_{1}+y_{2} u_{1}+z_{2} x_{1}+z_{3}, \\
u_{3} & =x_{1} u_{2}-y_{1} z_{2}+z_{1} y_{2}+u_{1} x_{2}, & x_{2} y_{2} & =-y_{2} x_{2}+z_{2} u_{2}-u_{2} z_{2}, \\
u_{2} x_{1} & =-x_{2} u_{1}-y_{2} z_{1}-z_{2} y_{1}, & x_{2} z_{2}=-y_{2} u_{2}-z_{2} x_{2}+u_{2} y_{2}, \\
u_{2} z_{1} & =-x_{2} y_{1}-y_{2} x_{1}-z_{2} u_{1}+y_{3}, & x_{2} u_{2}=-y_{2} z_{2}-z_{2} y_{2}-u_{2} x_{2} .
\end{array}
$$

The $\mathbb{k}$-coalgebra structure is given as follows:

$$
\begin{aligned}
\Delta\left(x_{k}\right) & =x_{k} \otimes 1+y_{k} \otimes x_{1}+u_{k} \otimes x_{2}+z_{k} \otimes x_{3}, \\
\varepsilon\left(x_{k}\right) & =0, \quad k=1,2,3, \\
\Delta\left(y_{k}\right) & =y_{k} \otimes y_{1}+u_{k} \otimes y_{2}+z_{k} \otimes y_{3}, \quad k=1,2,3, \\
\varepsilon\left(y_{k}\right) & =0, \quad k=2,3, \quad \varepsilon\left(y_{1}\right)=1, \\
\Delta\left(z_{k}\right) & =y_{k} \otimes z_{1}+u_{k} \otimes z_{2}+z_{k} \otimes z_{3}, \quad k=1,2,3, \\
\varepsilon\left(z_{k}\right) & =0, \quad k=1,2, \quad \varepsilon\left(z_{3}\right)=1, \\
\Delta\left(u_{k}\right) & =y_{k} \otimes u_{1}+u_{k} \otimes u_{2}+z_{k} \otimes u_{3}, \quad k=1,2,3, \\
\varepsilon\left(u_{k}\right) & =0, \quad k=1,3, \quad \varepsilon\left(u_{2}\right)=1 .
\end{aligned}
$$

Moreover $A$ is a left $\mathscr{L}_{m}(A)$-comodule algebra with coaction $\lambda: A \rightarrow \mathscr{L}_{m}(A) \otimes A$ defined by

$$
\begin{aligned}
\lambda\left(1_{A}\right) & =1_{\mathscr{L}(A)} \otimes 1_{A}, \\
\lambda(\mathfrak{i}) & =x_{1} \otimes 1+y_{1} \otimes \mathfrak{i}+u_{1} \otimes \mathfrak{j}+z_{1} \otimes \mathfrak{i j}, \\
\lambda(\mathfrak{j}) & =x_{2} \otimes 1+y_{2} \otimes \mathfrak{i}+u_{2} \otimes \mathfrak{j}+z_{2} \otimes \mathfrak{i j} .
\end{aligned}
$$

Of course, we have $\lambda(\mathfrak{i j})=\lambda(\mathfrak{i}) \lambda(\mathfrak{j})=x_{3} \otimes 1+y_{3} \otimes \mathfrak{i}+u_{3} \otimes \mathfrak{j}+z_{3} \otimes \mathfrak{i j}$.

ExAmple 2.0.2. Assume that $A=R^{n}$, the obvious $R$-ring attached to the free $R$-module of rank $n$. One can easily check, using (23) and Proposition 1.3.6, that $\mathscr{L}_{m}(A)$ is a left $R$-bialgebroid generated as a ring by the image of $R^{\mathrm{e}}$ and a set of $R^{\mathrm{e}}$-invariant elements $\left\{x_{i j}\right\}_{1 \leq i, j \leq n}$ with relations

$$
\begin{aligned}
x_{i i}^{2} & =x_{i i}, \quad \text { for all } i=1,2, \cdots, n, \\
x_{j i} x_{k i} & =0, \quad \text { for all } j \neq k, \text { and } i, j, k=1,2, \cdots, n, \\
\sum_{i=1}^{n} x_{i j} & =1, \quad \text { for all } j=1,2, \cdots, n .
\end{aligned}
$$

Its structure of $R$-coring is given by the following comultiplication and counit

$$
\begin{aligned}
\Delta\left(x_{i j}\right) & =\sum_{k=1}^{n} x_{i k} \otimes_{R} x_{k j}, \quad \text { for all } i, j=1,2, \cdots, n ; \\
\varepsilon\left(x_{i j}\right) & =\delta_{i j}, \quad \text { (Kronecker delta) for all } i, j=1,2, \cdots, n .
\end{aligned}
$$


Let us denote by $\left\{e_{i}\right\}_{1 \leq i \leq n}$ the canonical basis of ${ }_{R} A$. Then $A$ is a left $\mathscr{L}_{m}(A)$-comodule ring via the coaction $\lambda: A \rightarrow \mathscr{L}_{m}(A) \otimes_{R} A$ defined by

$$
\lambda\left(e_{i}\right)=\sum_{j=1}^{n} x_{i j} \otimes_{R} e_{j}, \quad \forall i=1, \cdots, n .
$$

EXAMPLE 2.0.3. Let $A=R \oplus R t$ be the trivial generalized $R$-ring i.e. the $R$-ring which is free as left $R$-module with basis $1=(1,0)$ and $\mathfrak{t}=(0, t)$ such that $\mathfrak{t}^{2}=0$. Using (23) and Proposition 1.3.6, we can easily check that $\mathscr{L}_{m}(A)$ is a left $R$-bialgebroid generated by the image of $R^{\mathrm{e}}$ and two $R^{\mathrm{e}}$-invariant elements $\{x, y\}$ subject to the relations $x y+y x=0$, $x^{2}=0$. The comultiplication and counit of its underlying $R$-coring are given by

$$
\begin{aligned}
& \Delta(x)=x \otimes_{R} 1+y \otimes_{R} x, \quad \varepsilon(x)=0, \\
& \Delta(y)=y \otimes_{R} y, \quad \varepsilon(y)=1 .
\end{aligned}
$$

$A$ is a left $\mathscr{L}_{m}(A)$-comodule ring with coaction $\lambda: A \rightarrow \mathscr{L}_{m}(A) \otimes_{R} A$ defined by

$$
\lambda\left(1_{A}\right)=1_{\mathscr{L}_{m}(A)} \otimes_{R} 1_{A}, \quad \lambda(\mathfrak{t})=x \otimes_{R} 1_{A}+y \otimes_{R} \mathfrak{t} .
$$

EXAMPLE 2.0.4. Let $A$ be the trivial crossed product of $R$ by the cyclic group $\mathcal{G}_{n}$ of order $n$. We know that ${ }_{R} A$ is the left free module with basis $\mathcal{G}_{n}$. It is easily checked, using (23) and Proposition 1.3.6, that if $n=2$, then $\mathscr{L}_{m}(A)$ is an $R$-bialgebroid generated as an $R^{\mathrm{e}_{-}}$ ring by two $R^{\mathrm{e}}$-invariant elements $x, y$ subject to the relations $x y+y x=0$ and $1=x^{2}+y^{2}$. The comultiplication and counit of the underlying $R$-coring structure are given by

$$
\Delta(x)=x \otimes_{R} 1+y \otimes_{R} x, \quad \Delta(y)=y \otimes_{R} y, \quad \varepsilon(x)=0, \quad \varepsilon(y)=1 .
$$

For $n>2$, we can prove that $\mathscr{L}_{m}(A)$ is an $R^{\mathrm{e}}$-ring generated by the $R^{\mathrm{e}}$-invariant elements $x_{(k, l)}$ with $(k, l) \in\left(\mathbb{Z}_{n} \backslash\{0\}\right) \times \mathbb{Z}_{n}$ subject to the following relations:

$$
\begin{gathered}
x_{(k, l)}=\sum_{s=0}^{n-1} x_{(t, l-s)} x_{(k-t, s)}, \forall(k, l) \in\left(\mathbb{Z}_{n} \backslash\{0,1\}\right) \times \mathbb{Z}_{n}, \forall t \in \mathbb{Z}_{n} \backslash\{0\} \text { with } t<k, \\
x_{(1, l)}=\sum_{s=0}^{n-1} x_{(n-t, l-s)} x_{\left(n-t^{\prime}, s\right)}, \forall l \in \mathbb{Z}_{n}, \forall t, t^{\prime} \in \mathbb{Z}_{n} \backslash\{0\}, \text { with } t+t^{\prime}=n-1,
\end{gathered}
$$

and

$$
1=\sum_{s=0}^{n-1} x_{(t, n-s)} x_{\left(t^{\prime}, s\right)}, \forall t, t^{\prime} \in \mathbb{Z}_{n} \backslash\{0\}, \text { with } t+t^{\prime}=0,
$$

where the $\operatorname{ring} \mathbb{Z}_{n}$ is endowed with the canonical ordering $0<1<\cdots<n-1$. The comultiplication and counit of its underlying $R$-coring structure are given by

$$
\Delta\left(x_{(k, l)}\right)=\sum_{s=0}^{n-1} x_{(k, s)} \otimes_{R} x_{(s, l)}, \quad \varepsilon\left(x_{(k, l)}\right)=\delta_{k, l}, \quad \forall(k, l) \in\left(\mathbb{Z}_{n} \backslash\{0\}\right) \times \mathbb{Z}_{n} .
$$

The left comodule ring structure of $A$ is given by the following coaction. Consider $\left\{\mathfrak{g}^{k}\right\}_{0 \leq k \leq n-1}$ the basis of the free module ${ }_{R} A$, where $\mathfrak{g}$ denotes the generating element of $\mathcal{G}_{n}$ which we 
identify with its image in $A$ by using the canonical injection. The coaction is then given by $\lambda: A \rightarrow \mathscr{L}_{m}(A) \otimes_{R} A$ sending

$$
\lambda\left(1_{A}\right)=1_{\mathscr{L}_{m}(A)} \otimes_{R} 1_{A}, \quad \lambda\left(\mathfrak{g}^{k}\right)=\sum_{l=0}^{n-1} x_{(k, l)} \otimes_{R} \mathfrak{g}^{l}, \forall k \in\left(\mathbb{Z}_{n} \backslash\{0\}\right) .
$$

\section{REFERENCES}

[1] T. Brzeziński and G. Militaru, Bialgebroids, $\times_{R}$-Bialgebras and duality. J. Algebra, 251 (2002), 279294.

[2] T. Brzeziński and R. Wisbauer, Corings and comodules, LMS, vol. 309, Cambridge University Press, 2003.

[3] G. Böhm, Hopf algebroids. Handbook of algebra. Vol. 6, 173-235. Elsevier/North-Holland, Amsterdam, 2009.

[4] A. Bruguières, Théorie tannakienne non commutative, Commun. in Algebra 22 (1994), 5817-5860.

[5] P. Deligne, Catégories tannakiennes. In The Grothendieck Festschrift (P. Cartier et al., eds), Progr. math., 87, vol. II, Birkhäuser, Boston, MA. 1990, pp. 111-195.

[6] N. Kowalzig, Hopf algebroids and their cyclic theory, Ph. D. thesis, Universiteit Utrecht and Universiteit van Amsterdam, 2009.

[7] Jiang-Hua Lu, Hopf algebroids and quantum groupoids. Int. J. Math. 7 (1996), 47-70

[8] S. Mac Lane, Categories for the working mathematician. Second edition. Graduate Texts in Mathematics, 5. Springer-Verlag, New York, 1998.

[9] D. C. Ravenel, Complex Cobordism and Stable Homotopy Groups of Spheres. Pure and Applied Mathematics Series, Academic Press, San Diego, 1986.

[10] P. Schauenburg, Bialgebras over noncommutative rings and a structure theorem for Hopf bimodules. App. Catg. Struct. 6 (1998), 193-222.

[11] P. Schauenburg, Duals and doubles of quantum groupoids ( $\times_{R}$-Hopf algebras), New trends in Hopf algebra theory (La Falda, 1999), Contemp. Math., vol. 267, Amer. Math. Soc., Providence, RI, 2000, pp. 273-299.

[12] M. Sweedler, Groups of simple algebras. I. H. E. S. Publ., 44 (1975), 79-189.

[13] M. Sweedler, The predual theorem to the Jacobson-Bourbaki theorem, Trans. Amer. Math. Soc. 213 (1975), 391-406.

[14] M. Takeuchi, Groups of algebras over $A \otimes \bar{A}$, J. Math. Soc. Japan 29 (1977), 459-492.

[15] D. Tambara, The coendomorphism bialgebra of an algebra, J. Fac. Sci. Univ. Tokyo Sect. IA Math. 37 (1990), no. 2, 425-456.

University of Turin, Department of Mathematics "Giuseppe Peano", via Carlo Alberto 10, I-10123 Torino, ItAly

E-mail address: alessandro.ardizzoni@unito.it

$U R L$ : http://www.unife.it/utenti/alessandro.ardizzoni

Universidad de Granada, Departamento de Álgebra. Facultad de Educación y Humanidades de Ceuta. El Greco N. 10. E-51002 Ceuta, Spain

E-mail address: kaoutit@ugr.es

University of Ferrara, Department of Mathematics, Via Machiavelli 35, Ferrara, I44121, ITALY

E-mail address: men@unife.it

$U R L:$ http://www. unife.it/utenti/claudia.menini 